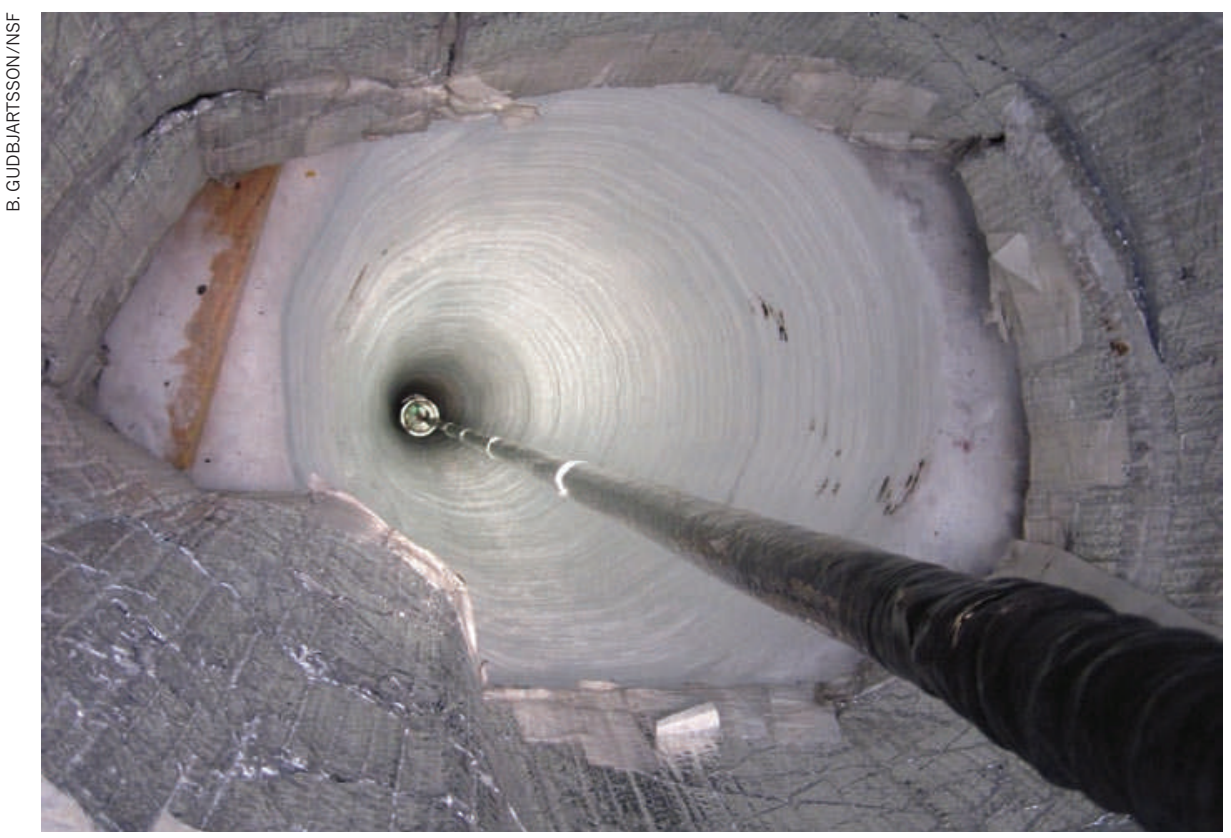

One of the detectors of the neutrino 'telescope' is lowered into place. ASTROPHYSICS

\section{IceCube completed}

\section{A neutrino observatory encased in ice at the South Pole could spot new physics.}

\section{BY ADAM MANN}

$\mathrm{O}$ $\mathrm{n}$ the day that researchers lowered the final detector of the IceCube Neutrino Observatory into a 2.5-kilometre-deep hole, the Antarctic sun was nearly as high as it gets and the temperature a balmy $-23^{\circ} \mathrm{C}$. "It is quite warm," reported team member Albrecht Karle, a physicist at the University of Wisconsin in Madison. Just a week before the 18 December event, he adds, temperatures had averaged about $10^{\circ} \mathrm{C}$ colder.

Karle and his collaborators aren't complaining. The deep Antarctic cold has created an ideal natural medium for detecting high-energy neutrinos. At a depth of 1.5 kilometres below the surface, the sheer weight of the overlying layers at the South Pole keeps the Antarctic

$\vec{z}$ ice sheet free of air bubbles and thus perfectly clear. Within its dark, transparent depths, even a faint flash of light can be spotted at some distance - including the kind of flash signalling that a fast-moving neutrino has hit an oxygen atom sitting in the ice and produced a muon.

The detector, a basketball-sized optical sensor, covered in the collaborators' signatures, was the 5,160 th to be placed since construction began on IceCube in 2005. Since then, every Antarctic summer, researchers have used a jet of near-boiling water to drill holes in the ice. At every hole, a kilometre-long string of detectors was lowered down and the hole allowed to refreeze.

Now complete, at a cost of about US $\$ 271$ million, IceCube monitors a cubic kilometre of ice - the size required for the experiment to have a realistic chance of spotting its rare and elusive quarry. But as the instrument has grown, so has its scope. Over the years, the team has realized that IceCube might also shed light on a broader range of questions, including an understanding of physics beyond the standard model.

"The most interesting things we're doing now, we didn't think of when it was proposed," says Francis Halzen, a physicist at the University of Wisconsin in Madison and the IceCube collaboration's principal investigator.

After photons, neutrinos are the most common particle in the Universe - trillions stream

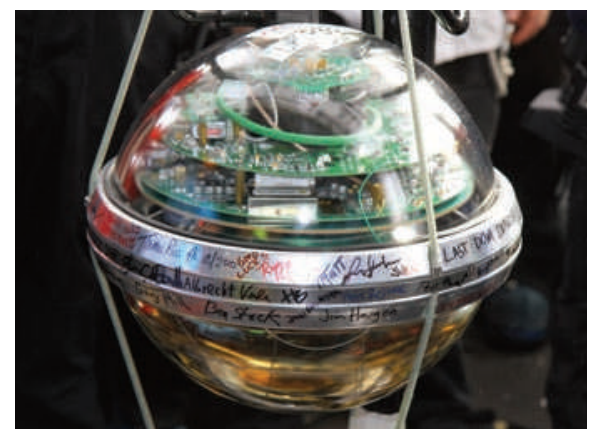

The final detector: signed, sealed and delivered. through your body every second. They are created in the nuclear-reactor centres of stars such as the Sun and by a variety of other objects in space. And because they are electrically neutral, unaffected by magnetic fields that permeate space, their straight-line trajectories point back to their sources.

If high-energy neutrinos turn up in IceCube's data as expected, they will provide astronomers with information they could not get from photons, says Eli Waxman, an astrophysicist at the Weizmann Institute of Science in Rehovot, Israel. One mystery it could crack is the source of ultra-high-energy cosmic rays (UHECRs), a poorly understood class of particles that carry billions of times the energy achievable in today's most powerful particle accelerators.

The two candidates currently suspected of generating UHECRs - active galactic nuclei and $\gamma$-ray bursts - are both powered by the accretion of mass onto a black hole. Yet the process by which these objects convert gravitational energy to radiation and particles is one of the fundamental open questions in astrophysics, says Waxman. IceCube could simultaneously solve the UHECR puzzle and provide qualitatively new information on the way matter behaves under strong gravity.

IceCube's size also makes it ideal for understanding completely new physical phenomena, says Bill Louis, a physicist at Los Alamos National Laboratory in New Mexico and co-spokesman for the neutrino experiment MiniBooNE at Fermilab in Batavia, Illinois. As neutrinos travel, they may oscillate between three known types - electron, muon and tau - but results published last October suggest that MiniBooNE may have observed muon antineutrinos turning into a fourth type, called a sterile neutrino (A. A. Aguilar-Arevalo et al. Phys. Rev. Lett. 105, 181801; 2010). The energy range over which IceCube searches makes it perfectly configured to discern this fluctuation, says Louis, which means that it might be able to confirm or overturn the strange MiniBooNE result, he adds.

IceCube might also help to reveal the nature of dark matter, an invisible component of the Universe that may consist of one or more types of weakly interacting particles (WIMPs). In some models, WIMPs are predicted to accumulate at the Sun's core. Such a concentration would increase the odds of dark-matter particles colliding with one another and annihilating to produce high-energy neutrinos, says Robert Stokstad, a physicist and project director for IceCube at Lawrence Berkeley National Laboratory in California. If IceCube could find these neutrinos and confirm that they originate in the Sun, it would be the first direct detection of dark matter, he says.

Waxman says that the most exciting possibility is that IceCube will detect something entirely unexpected. "In the most basic respect," Waxman says, "IceCube is providing us with a new way to look at the Universe." - 